\title{
Evolution of head-shaft angle and neck-shaft angle in childhood
}

\author{
Pieter Van Geel, Wilfried Cools, Armand Laumen
}

From the Department of Orthopaedic Surgery, Universitair Ziekenhuis Brussel, Brussels, Belgium

The current retrospective study investigates the natural evolution of head-shaft angle (HSA) and neck-shaft angle (NSA) in childhood. It is not known if a high HSA in early childhood leads to a high HSA in adulthood. This study aims to characterize the evolution of HSA and compares it with the more commonly known NSA in healthy children.

We measured radiographs of 84 different healthy hips of children between 3 and $\mathbf{1 4 . 5}$ years old who underwent at least 2 radiographs of the pelvis, corresponding to $\mathbf{2 8 6}$ measurements. We used a linear mixed model to determine the covariance between random intercept and slope while allowing each individual hip to change over time.

The covariance for HSA between random intercept and random slope was $\mathbf{- 4 . 2 6 2}(\mathbf{p}<\mathbf{0 . 0 0 1})$, corresponding to a high negative correlation of $\mathbf{- 0 . 7 1 7}$, for NSA $\mathbf{- 2 . 7 5 4}$ $(p=0.031)$ or a high negative correlation of $\mathbf{- 0 . 7 7 9}$. HSA and NSA were strongly correlated, a value of 0.736 ( $p<0.001)$ was measured.

The high negative correlation for random intercept and random slope means that the higher the initial value (intercept), the steeper the decline (slope). Therefore HSA decreases faster in hips with high HSA at an early age. Hips with high HSA in early childhood do not necessarily lead to hips with high HSA in adulthood. Our results may aid in future clinical decision making in patients with developmental dysplasia of the hip (DDH) with high HSA in particular.

Conflict of Interest: P. Van Geel, W. Cools, A. Laumen declare that they have no conflict of interest.

Funding statement: No benefits in any form have been received or will be received from a commercial party related directly or indirectly to the subject of this article.

Ethical approval: This article does not contain any studies with human participants or animals performed by any of the authors.
Keywords : proximal femoral anatomy; head-shaft angle ; neck-shaft angle ; HSA ; NSA.

\section{INTRODUCTION}

The growth and development of the child's hip is a complicated process between a growing proximal femur, a growing acetabulum and the vasculature. To achieve a congruent joint, a profound and delicate interplay between the acetabulum and the proximal femur is crucial $(1,2)$.

A common hip abnormality is developmental dysplasia of the hip (DDH). DDH comprises the whole range of deformities in the growing hip including dislocations, subluxations, instability and dysplasia of the acetabulum and deformities of the proximal femur (3).

If conservative treatment for DDH is insufficient, pelvic and/or femoral osteotomies are sometimes

P. Van Geel' ${ }^{1}, \mathrm{MD}$, Resident Orthopaedic Surgeon

- W. Cools ${ }^{2}, \mathrm{PhD}$, Statistical Consultant

- Laumen ${ }^{1}, \mathrm{MD}$, Pediatric Orthopaedic Surgeon

${ }^{1}$ Department of Orthopaedic Surgery, Universitair Ziekenhuis Brussel, Brussels, Belgium.

${ }^{2}$ Interfaculty Center Data processing and Statistics, Vrije Universiteit Brussel, Brussels, Belgium.

Correspondence : P. Van Geel, Department of Orthopaedic Surgery, Universitair Ziekenhuis Brussel, Laarbeeklaan 101, Brussels, Belgium.

Email : pivangeel@gmail.com

- 2021, Acta Orthopædica Belgica.

Acta Orthopædica Belgica, Vol. 87 - 3 - 2021 
needed in addition to open reduction. Varus Derotation Osteotomy (VDRO) is an example of a femoral osteotomy that redirects the femoral head towards the centre of the acetabulum, while a pelvic osteotomy, for example Salter Innominate Osteotomy (SIO), corrects the acetabular dysplasia directly $(4,5)$.

It is still debatable if the initiation of DDH is primarily acetabular and/or femoral based and accordingly the proximal femoral anatomy is regarded by some as part of the disease $(6,7)$. The role that the proximal femoral anatomy plays on the acetabular development remains debated and is still under investigation (8). In young patients with $\mathrm{DDH}$, a high head-shaft angle (HSA) may be seen as an argument to prefer a femoral or varus osteotomy over an acetabular osteotomy. The underlying idea is that a high HSA in childhood will lead to a high HSA at the end of growth.

Over the last decade, HSA has regained interest as parameter to evaluate proximal femoral morphology since it was first introduced by W.O. Southwick et al. in 1967 (9). A high HSA is frequently associated with Cerebral Palsy (CP) and seems to be a possible risk factor for hip displacement in children with $\mathrm{CP}$ (10-14). Reference values in healthy children have been reported and Santili et al. found no significant difference according to chronological age (15). Three years later, the same research team found, in contrast to their previous findings, an inverse correlation of the HSA with chronological $(r=-0.57)$ and bone age $(r=-0.52)$. They proposed to consider HSA between 148 and 155 degrees to be normal (16). Van der List et al. measured the contralateral hips of patients with unilateral DDH and found a decrease of 2 degrees per year (13).

Several studies have demonstrated that HSA is an excellent parameter regarding inter- and intrarater reliability and that HSA is less dependent of rotation of hips than the more commonly used neck-shaft angle (NSA) (8,15). Intraclass Correlation Coefficients (ICC) of 0.92 (95\% Confidence Interval (CI) $0.87-0.96)$ and 0.99 (95\% CI 0.98-0.99) for inter- and intra-rater reliability respectively were found by Hermanson et al. $(10,17,18)$.

As mentioned above, there is only limited data on the normal range and evolution of HSA and data are often based on small study populations or age categories. HSA is a reliable parameter to assess the proximal femoral geometry and it could be useful to know more about this for individual patients. We retrospectively analysed pelvic radiographs at multiple ages per single individual hip to determine whether hips with high HSA evolve differently than hips with moderate or low HSA in typically developing children. A possible correlation between the evolution of HSA and NSA was investigated.

\section{PATIENTS AND METHODS}

This study was reviewed and approved by the Medical Ethical Committee of our institution (no. 2016/126).

We selected patients from a digital database with at least two pelvic radiographs taken between the age of 3 to 16 years. These radiographs were taken from June 2005 until September 2017. Medical records of the 291 selected patients, corresponding to 1016 radiographs, were reviewed. Patients with a disease that could potentially affect the proximal femur, for example Cerebral Palsy, were excluded. From patients with Legg-Calve-Perthes disease (LCPD), DDH, Slipped Capital Femoral Epiphysis (SCFE) or fractures of the proximal femur, only the unaffected side was used for measurements. When there was any prior surgery to the proximal femur, the operated side was also excluded. Patients were also excluded if there wasn't at least 1 year between their first and last radiograph of the pelvis. Finally, radiographs showing closed physis or only a limited part of the femur, too short for accurate measurement, were excluded. Application of this criteria resulted in a total of 71 patients or 84 different hips remaining in this study. From 13 patients both hips could be included. Measurements were performed on 255 radiographs or 286 hips.

\section{Head-Shaft Angle}

We used the method of measurement as described by Southwick et al. for the determination of the HSA (Figure 1). Line $A$ is drawn across the base of the epiphysis, connecting the superior and inferior margins of the epiphysis. Line $B$ is drawn 


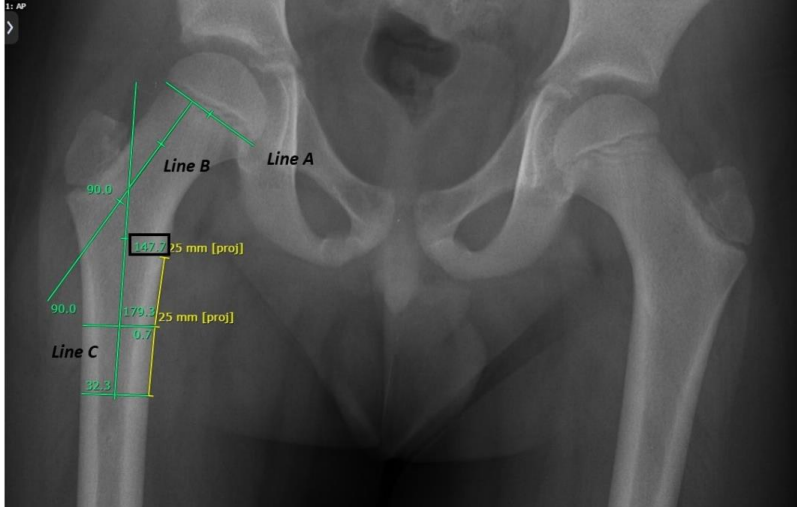

Fig. 1. - Anteroposterior radiograph of the pelvis. Method of measurement of the antero-posterior Head-Shaft Angle as described by Southwick et al. Line $A$ is drawn across the base of the epiphysis, connecting the superior and inferior margins of the epiphysis. Line $B$ is drawn at a 90-degree angle to Line $A$. Line $C$ represents the femoral shaft axis or Femoral Long Axis (FLA). The FLA was drawn through the midpoint of the shaft at 25 and 50 millimetres below the lesser trochanter or, if this was not possible due to the length of the femoral shaft on the radiograph, a line though the middle of the most inferior part of the shaft on the radiograph and a line touching the medial border of the greater trochanter. The angle between Line $C$ and Line $B$ is called the Head-Shaft Angle (HSA) $(9,19)$.

at a 90 -degree angle to Line $A$. The angle between Line $C$ and Line $B$ is called the Head-Shaft Angle (HSA) (9). Line $C$ represents the femoral shaft axis or Femoral Long Axis (FLA). The FLA was not defined in the original article by Southwick et al. We defined the FLA in accordance with the study of Clark et al. A line was drawn through the midpoint of the shaft at 25 and 50 millimetres below the lesser trochanter or, if this was not possible due to the length of the femoral shaft on the radiograph, a line through the middle of the most inferior part of the shaft on the radiograph and touching the medial border of the greater trochanter (19).

\section{Neck-Shaft Angle}

We measured the NSA as follows. The Femoral Neck Axis (FNA) is a line crossing the femoral head centre (HC) and the femoral neck centre (NC) [(20)]. The HC was determined using Mose circles. The NC was defined by the method of Müller; the midpoint on a line across the narrowest part of the femoral neck $(21,22)$. The definition of Femoral Long

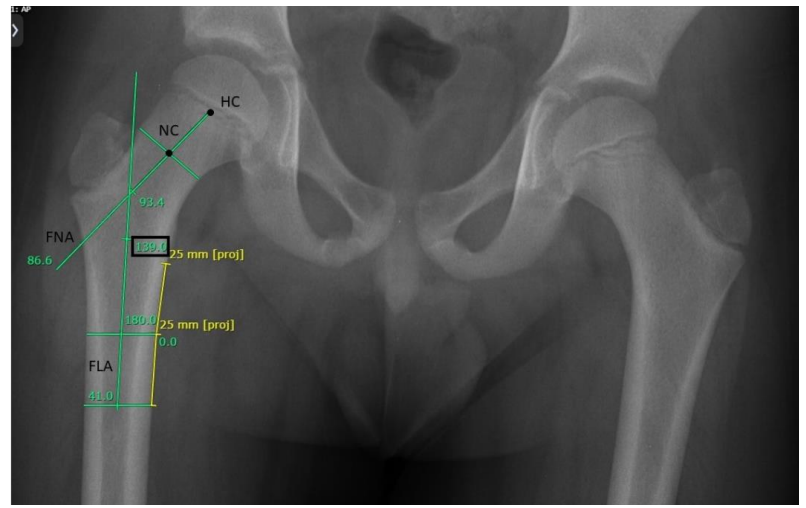

Fig. 2. - Anteroposterior radiograph of the pelvis. Method of measurement of the Neck-Shaft Angle as suggested by Boese et al. The Femoral Neck Axis (FNA) is a line crossing the femoral head centre (HC) and the femoral neck centre (NC) (20). The $\mathrm{HC}$ was determined using Mose circles. The $\mathrm{NC}$ was defined by the method of M.E. Müller ; the midpoint on a line across the narrowest part of the femoral neck $(21,22)$. The Femoral Long Axis (FLA) was drawn through the midpoint of the shaft at 25 and 50 millimetres below the lesser trochanter or, if this was not possible due to the length of the femoral shaft on the radiograph, a line though the middle of the most inferior part of the shaft on the radiograph and a line touching the medial border of the greater trochanter. The angle between FNA and FLA represents the NSA (19).

Axis (FLA) is mentioned before. The angle between FNA and FLA represents the NSA (Figure 2).

\section{Statistical Analysis}

All statistical analyses were performed using $\mathrm{R}$ version 3.5.1 (2018-07-02).

To assess whether HSA and NSA in hips with high HSA or NSA at young age decrease faster, a linear mixed model was used with focus on the covariance between the intercept (the initial value) and the slope (the change over time). Such a model can address the relation between the initial value and the change over time without having to resort to suboptimal subgroup analyses. Furthermore, the linear mixed model evaluates the average change over time while accounting for the correlation implied by performing repeated measurements within the same hip. Various models were evaluated and compared for both HSA and NSA including pairwise interactions and higher order polynomials over time. Because consecutive measurements 


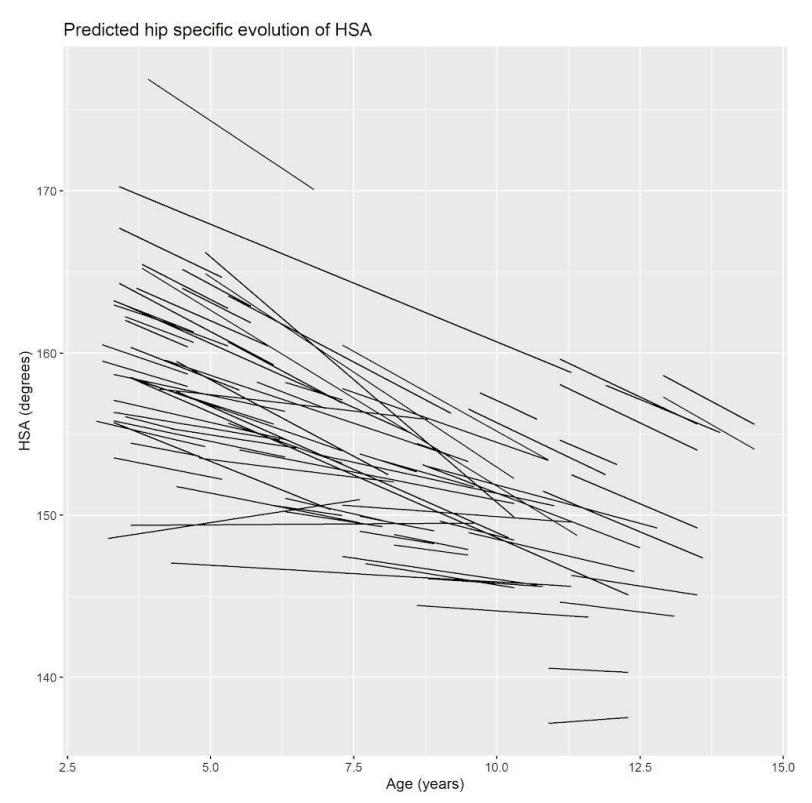

Fig. 3. - Predicted hip specific evolution of HSA according to age. Vertical axis represents HSA (degrees) and horizontal axis age (years).

tend to relate more strongly with smaller time intervals, an autoregressive correlation (type AR1) is included. During the model building, we used primarily likelihood ratio tests with Restricted Maximum Likelihood (REML) estimation for testing the variances and covariances and Maximum Likelihood (ML) estimation for testing the fixed effects. To compare the HSA and NSA, a Pearson bivariate correlation was applied. P-values $<0.05$ were considered statistically significant.

\section{RESULTS}

The predicted hip specific evolutions over age, based on the 286 measurements for 84 different hips, are visualised for HSA (Figure 3) and NSA (Figure 4). The male/female ratio was 33:38 for the 71 patients and 40:44 for the 84 different hips (inclusion of both hips of 7 male patients and of 6 female patients). The youngest patient was three years old and the oldest 14.5 years at the time of the radiograph (x-axis Figure 3 and 4 ). The mean age of the measured hips was 7.4 years (standard deviation (SD) 2.9). The HSA ranged from 133.8 to 177.8 (y-axis Figure 3) with a mean of 154.1 degrees (SD

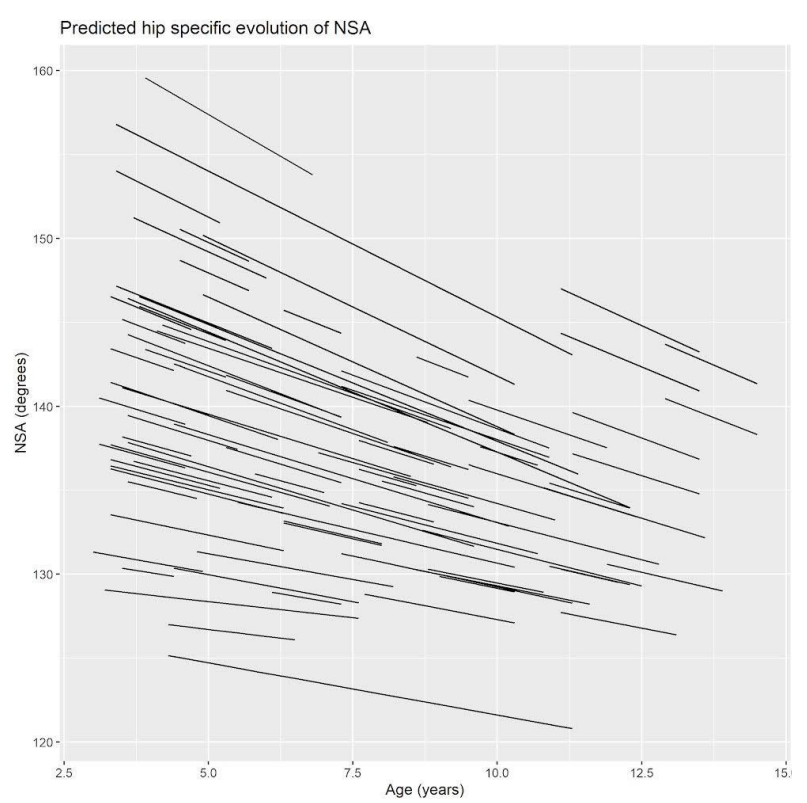

Fig. 4. - Predicted hip specific evolution of NSA according to age. Vertical axis represents NSA (degrees) and horizontal axis age (years).

7.2) and the NSA from 113.9 to 165.6 (y-axis Figure 4) with a mean of 136.6 degrees (SD 8.7). The mean HSA and NSA decreased consistently per age year during the first years (Table I).

Analysis of the HSA showed that the mean angle at age 3 was 159.7 degrees (95\% CI 157.6 to 161.7) and in average the angle decreased with 1.1 degrees each year $(95 \% \mathrm{CI}-1.5$ to -0.8$)$. The initial HSA at age 3 (intercept) differed substantially between different hips (SD 6.392) $(p<0.001)$. Also the evolution of HSA over time (slope) showed significant heterogeneity (SD 0.930) ( $<<0.001$ ). The covariance for intercept and slope was -4.262 $(p<0.001)$ corresponding to a correlation of -0.717 .

For NSA, results for the mean angle at age 3 was $140.6(95 \%$ CI 138.1 to 143.3$)$ and in average the angle decreased with 1.1 degrees per year $(95 \% \mathrm{CI}$ -1.5 to -0.7$)$. The standard deviations were 7.474 $(\mathrm{p}=0.064)$ and $0.473(\mathrm{p}<0.001)$ for intercept and slope respectively. The covariance between both parameters showed significance too. The covariance of $-2.754(p=0.031)$ implied a correlation of -0.779 . The measurements of HSA and NSA were strongly correlated $(\mathrm{r}=0.736)(\mathrm{p}<0.001)$. 
Table I. - Results of mean HSA and NSA per age year and corresponding range (standard deviations given between brackets)

\begin{tabular}{|l|c|c|c|c|c|}
\hline Age (years) & $\mathrm{n}$ hips & HSA $\left(^{\circ}\right)$ mean & HSA $\left(^{\circ}\right)$ range & NSA $\left(^{\circ}\right)$ mean & NSA $\left(^{\circ}\right)$ range \\
\hline 3 & 36 & $160.0(6.4)$ & 147.3 to 177.8 & $141.2(10.4)$ & 121.1 to 165.6 \\
\hline 4 & 44 & $158.2(7.0)$ & 143.2 to 170.6 & $140.5(10.2)$ & 122.0 to 158.7 \\
\hline 5 & 28 & $157.1(8.0)$ & 140.7 to 175.1 & $138.8(9.9)$ & 121.6 to 157.4 \\
\hline 6 & 29 & $154.8(5.9)$ & 145.9 to 176.4 & $134.4(7.3)$ & 124.4 to 157.4 \\
\hline 7 & 33 & $151.6(4.5)$ & 144.7 to 163.3 & $134.8(5.6)$ & 121.2 to 150.3 \\
\hline 8 & 36 & $151.0(4.9)$ & 141.5 to 162.5 & $133.2(7.5)$ & 115.4 to 150.7 \\
\hline 9 & 19 & $151.8(4.5)$ & 145.6 to 159.4 & $136.1(4.9)$ & 126.0 to 145.5 \\
\hline 10 & 21 & $148.7(6.0)$ & 133.8 to 157.3 & $132.9(5.4)$ & 124.4 to 140.6 \\
\hline 11 & 21 & $150.4(6.5)$ & 141.4 to 161.5 & $133.7(9.3)$ & 113.9 to 148.4 \\
\hline 12 & 10 & $148.7(7.7)$ & 136.2 to 159.6 & $133.8(5.5)$ & 126.1 to 143.8 \\
\hline 13 & 7 & $151.4(7.7)$ & 143.3 to 161.6 & $136.8(8.6)$ & 125.9 to 148.1 \\
\hline 14 & 2 & $157.4(3.7)$ & 154.7 to 160.0 & $142.7(5.0)$ & 139.2 to 146.2 \\
\hline Total/mean* & 286 & $154.1(7.2)$ & 133.8 to 177.8 & $136.6(8.7)$ & 113.9 to 165.6 \\
\hline
\end{tabular}

*The Total/mean is the mean of all the measured values and not of the mean values per age.

\section{DISCUSSION}

Our study demonstrates that a high HSA in early childhood does not result in a high HSA in adulthood. The correlation coefficient for intercept and slope of -0.717 implies that hips with a high HSA at early age (corresponding to a high intercept) decrease faster to a lower HSA or have a more negative slope (p-values $<0.001$ ). For the NSA, which is strongly correlated with the HSA, the same conclusions are suggested based on the correlation coefficient of -0.779 , but data were too limited to conclude that the NSA evolved differently for different hips ( $p$-value $=0.064$ for intercept). Regarding the size of correlation coefficients, values between -0.70 to -0.90 are generally considered high negative correlations (23).

The advantage of the statistical method used in this study is that there is no need to perform subgroup analysis. The linear mixed model evaluates the average change of the HSA and NSA angles, and more importantly how these angles vary in the population and how the evolution relates to the initial value.

Our findings for the evolution of HSA are similar to the results of van der List et al. in the inverse correlation between HSA and age. They found that $\mathrm{HSA}=171.4^{\circ}-2.0^{\circ} \mathrm{x}$ age (years) where we obtained $\mathrm{HSA}=159.7^{\circ}-1.1^{\circ} \mathrm{x}$ age (years). Our less negative regression coefficient can be explained by a lower intercept and the wider age range of the study population to higher ages (13).

In general, we observed that HSA and NSA evolve in a very similar way and are strongly correlated (correlation coefficient, 0.736 (p < $0.001)$ ). The regression coefficients are both -1.1 in degrees per year and the correlation coefficient for intercept and slope of HSA and NSA (-0.717 and -0.779 respectively) are also close to each other. Note that Lee et al. found a quite similar correlation coefficient of 0.718 for HSA and NSA, when measuring hips of 348 patients with CP (24).

Even though the NSA is one of the most commonly used parameters to assess the geometry of the proximal femur, it has some shortcomings. Firstly, the method of measurement varies significantly in the literature. Secondly, the NSA is influenced by the femoral ante-and retroversion and hip rotation $(20,25)$. Thirdly, it does not take into account the proximal femoral epiphysis or physis (24). The HSA, however, is based on the orientation of the proximal femoral physis and is less dependent of hip rotation. Further, several studies showed an excellent intra-and inter-observer variability, on which we decided to rely on a single measurement by the same examiner $(10,13,18)$. For the above mentioned reasons, we advise the use of HSA in assessing the geometry of the proximal femur in children. 
To account for the use of contralateral hips of patients with diseases possibly affecting the proximal femur, the medical history and radiographs were reviewed by a senior orthopaedic surgeon and pediatric radiologist before measurement of the contralateral side.

Our study suggests that a high HSA in young patients does not automatically lead to a valgus hip in adulthood and therefore is not a deformity that needs to be addressed in all patients. This may be an argument in favour of an acetabular osteotomy where perhaps a femoral osteotomy would be chosen in young patients with DDH in the presence of a degree of proximal femoral valgus, in whom previous treatment did not achieve a sufficient improvement (5). A remark here is that we showed that healthy hips with high HSA decreased faster to lower values, but whether this is applicable for hips with DDH is unclear. Sugano et al. compared the morphometry of the femur in patients with DDH and control patients. They found that the femora with DDH had 10 to 14 degrees more anteversion but no significantly different NSA was observed (6). Since results for NSA and HSA were quite similar, we expect that also the HSA, would not be significantly different and hips with DDH would follow the same trend as healthy hips.

Further research should attempt to recruit more children at ages above 11 years old to establish how the evolution progresses towards adulthood. While in our study there were children with ages up to 14.5 years old, these were underrepresented. The importance of large study populations in establishing reference values is shown once more in the study of Beutel et al. In their study of the femoral anatomy in children, no correlation between NSA and age was found. They explained this inconsistency with existing literature with the fact that they included only 7 femurs under 8 years old (26).

In conclusion, our study demonstrates that HSA decreases faster in hips with high HSA at an early age. Hips with high HSA in early childhood do not necessarily lead to hips with high HSA in adulthood. Our results may aid in future clinical decision making in patients with DDH with high HSA in particular.

\section{Compliance with ethical standards}

The authors declare that there is no conflict of interest.

No benefits in any form have been received or will be received from a commercial party related directly or indirectly to the subject of this article.

This article does not contain any studies with human participants or animals performed by any of the authors.

\section{Author contributions}

P. Van Geel, MD (Study design, Study implementation, Performed measurements, Statistical analysis, Writing the paper)

W. Cools, PhD (Study design, Statistical analysis, Revision of manuscript, Approved the final manuscript)

A. Laumen, MD (Study design, Performed measurements, Revision of manuscript, Approved the final manuscript)

\section{REFERENCES}

1. Lee MC, Eberson CP. Growth and development of the child's hip. Orthop Clin North Am. 2006 ; 37(2) : 119-32, v.

2. Weinstein SL. Natural history of congenital hip dislocation (CDH) and hip dysplasia. Clin Orthop Relat Res. 1987 (225) : 62-76.

3. Kotlarsky P, Haber R, Bialik V, Eidelman M. Developmental dysplasia of the hip : What has changed in the last 20 years? World J Orthop. 2015 ; 6(11) : 886-901.

4. Böhm P, Brzuske A. Salter innominate osteotomy for the treatment of developmental dysplasia of the hip in children : results of seventy-three consecutive osteotomies after twenty-six to thirty-five years of follow-up. $J$ Bone Joint Surg Am. 2002 ; 84-A(2) : 178-86.

5. Spence G, Hocking R, Wedge JH, Roposch A. Effect of innominate and femoral varus derotation osteotomy on acetabular development in developmental dysplasia of the hip. J Bone Joint Surg Am. 2009 ; 91(11) : 2622-36.

6. Sugano N, Noble PC, Kamaric E, Salama JK, Ochi T, Tullos HS. The morphology of the femur in developmental dysplasia of the hip. J Bone Joint Surg Br. 1998 ; 80(4) : 711-9.

7. Mahieu P, Hananouchi T, Watanabe N, Claes P, Li H, Audenaert E. Morphological abnormalities of the femur in the dysplastic hip. Relation between femur en acetabulum. Acta Orthop Belg. 2018 ; 84(3) : 307-15. 
8. Moraleda L, Bravo C, Forriol F, Albiñana J. Does Orientation of the Femoral Head Affect Acetabular Development? An Experimental Study in Lamb. J Pediatr Orthop. $2019 ; 39(8): 416-21$.

9. Southwick WO. Osteotomy through the lesser trochanter for slipped capital femoral epiphysis. J Bone Joint Surg Am. 1967 ; 49(5) : 807-35.

10. Foroohar A, McCarthy JJ, Yucha D, Clarke S, Brey J. Head-shaft angle measurement in children with cerebral palsy. J Pediatr Orthop. 2009 ; 29(3) : 248-50.

11. Hermanson M, Hägglund G, Riad J, Wagner P. Headshaft angle is a risk factor for hip displacement in children with cerebral palsy. Acta Orthop. 2015 ; 86(2) : 229-32.

12. van der List JP, Witbreuk MM, Buizer AI, van der Sluijs JA. The prognostic value of the head-shaft angle on hip displacement in children with cerebral palsy. J Child Orthop. 2015 ; 9(2) : 129-35.

13. van der List JP, Witbreuk MM, Buizer AI, van der Sluijs JA. The head-shaft angle of the hip in early childhood : a comparison of reference values for children with cerebral palsy and normally developing hips. Bone Joint J. 2015 ; 97-B(9) : 1291-5.

14. Chougule S, Dabis J, Petrie A, Daly K, Gelfer Y. Is head-shaft angle a valuable continuous risk factor for hip migration in cerebral palsy? J Child Orthop. 2016 ; 10(6) : 651-6.

15. Santili C, de Assis MC, Kusabara FI, Romero IL, Sartini CM, Longui CA. Southwick's head-shaft angles : normal standards and abnormal values observed in obesity and in patients with epiphysiolysis. J Pediatr Orthop B. 2004 ; 13(4) : 244-7.

16. Damaceno FL, Santili C, Longui CA. Normal reference values of Southwick's anteroposterior angle in prepubertal and pubertal normal adolescents. J Pediatr Orthop B. 2007 ; 16(6) : 389-92.

17. Wynter M, Gibson N, Kentish M, Love S, Thomason P, Kerr Graham H. The Consensus Statement on Hip
Surveillance for Children with Cerebral Palsy : Australian Standards of Care. J Pediatr Rehabil Med. 2011 ; 4(3) : 183-95.

18. Hermanson M, Hägglund G, Riad J, Rodby-Bousquet E. Inter- and intra-rater reliability of the head-shaft angle in children with cerebral palsy. J Child Orthop. 2017 ; 11(4) : 256-62.

19. Clark JM, Freeman MA, Witham D. The relationship of neck orientation to the shape of the proximal femur. $J$ Arthroplasty. 1987 ; 2(2) : 99-109.

20. Boese CK, Dargel J, Oppermann J, Eysel P, Scheyerer MJ, Bredow J, et al. The femoral neck-shaft angle on plain radiographs : a systematic review. Skeletal Radiol. 2016 ; 45(1) : 19-28.

21. Høiseth A, Reikerås O, Fønstelien E. Evaluation of three methods for measurement of femoral neck anteversion. Femoral neck anteversion, definition, measuring methods and errors. Acta Radiol. 1989 ; 30(1) : 69-73.

22. Mueller M. The intertrochanteric osteotomy and pseudarthrosis of the femoral neck (Reprinted from Die Huftnahen Femurosteotomien, pg 113-119, 1957). Clinical Orthopaedics and Related Research. 1999(363) : 5-8.

23. Mukaka MM. Statistics corner : A guide to appropriate use of correlation coefficient in medical research. Malawi Med J. $2012 ; 24(3): 69-71$.

24. Lee KM, Kang JY, Chung CY, Kwon DG, Lee SH, Choi IH, et al. Clinical relevance of valgus deformity of proximal femur in cerebral palsy. J Pediatr Orthop. 2010 ; 30(7) : 720-5.

25. Boese CK, Frink M, Jostmeier J, Haneder S, Dargel J, Eysel P, et al. The Modified Femoral Neck-Shaft Angle : Age- and Sex-Dependent Reference Values and Reliability Analysis. Biomed Res Int. 2016 ; 2016 : 8645027.

26. Beutel BG, Girdler SJ, Collins JA, Otsuka NY, Chu A. Characterization of proximal femoral anatomy in the skeletally-immature patient. J Child Orthop. 2018 ; 12(2) : 167-72. 